Supporting Information

\title{
Estimation of lipoyllysine content in meat and its antioxidative capacity
}

Adrianna Kamińska and Grażyna Chwatko*

Faculty of Chemistry, Department of Environmental Chemistry, University of Lodz, 163 Pomorska Str., 90-236 Łódź, Poland

*Corresponding author. Email: grazyna.chwatko@ chemia.uni.lodz.pl , Phone: +48 426355843,

Fax: +48426355832 .

Table S1. The recommended amounts of proteases (subtilisin A and pronase E) added to the studied animal tissues to determine content LA and LLys.

\begin{tabular}{lcccc}
\hline \multicolumn{1}{c}{ Sample } & $\begin{array}{c}\text { Activity of } \\
\text { Pronase E }\end{array}$ & $\begin{array}{c}\text { Volume of } \\
\text { Pronase E }\end{array}$ & $\begin{array}{c}\text { Activity of } \\
\text { subtilisin A }\end{array}$ & $\begin{array}{c}\text { Volume of } \\
\text { subtilisin A }\end{array}$ \\
\hline LLys & & & & \\
Turkey heart & $9 \mathrm{U}$ & $10 \mu \mathrm{L}$ & $1 \mathrm{U}$ & $0.7 \mu \mathrm{L}$ \\
Calf heart & $9 \mathrm{U}$ & $10 \mu \mathrm{L}$ & $1 \mathrm{U}$ & $0.7 \mu \mathrm{L}$ \\
liver & $8 \mathrm{U}$ & $10 \mu \mathrm{L}$ & $2 \mathrm{U}$ & $1.5 \mu \mathrm{L}$ \\
kidney & $4 \mathrm{U}$ & $10 \mu \mathrm{L}$ & $6 \mathrm{U}$ & $4.4 \mu \mathrm{L}$ \\
Beef heart & $8 \mathrm{U}$ & $10 \mu \mathrm{L}$ & $2 \mathrm{U}$ & $1.5 \mu \mathrm{L}$ \\
liver & $24 \mathrm{U}$ & $10 \mu \mathrm{L}$ & $16 \mathrm{U}$ & $11.7 \mu \mathrm{L}$ \\
kidney & $36 \mathrm{U}$ & $10 \mu \mathrm{L}$ & $4 \mathrm{U}$ & $2.9 \mu \mathrm{L}$ \\
stomach & $8 \mathrm{U}$ & $10 \mu \mathrm{L}$ & $2 \mathrm{U}$ & $1.5 \mu \mathrm{L}$ \\
Chicken heart & $8 \mathrm{U}$ & $10 \mu \mathrm{L}$ & $2 \mathrm{U}$ & $1.5 \mu \mathrm{L}$ \\
liver & $5 \mathrm{U}$ & $10 \mu \mathrm{L}$ & $5 \mathrm{U}$ & $3.7 \mu \mathrm{L}$ \\
kidney & $4 \mathrm{U}$ & $10 \mu \mathrm{L}$ & $6 \mathrm{U}$ & $4.4 \mu \mathrm{L}$ \\
Pig heart & $8 \mathrm{U}$ & $10 \mu \mathrm{L}$ & $2 \mathrm{U}$ & $1.5 \mu \mathrm{L}$ \\
kidney & $12 \mathrm{U}$ & $10 \mu \mathrm{L}$ & $28 \mathrm{U}$ & $20.5 \mu \mathrm{L}$ \\
stomach & $8 \mathrm{U}$ & $10 \mu \mathrm{L}$ & $2 \mathrm{U}$ & $1.5 \mu \mathrm{L}$ \\
& & & & \\
LA & & & 10 & $2 \mathrm{U}$ \\
Turkey heart & $9 \mathrm{U}$ & $10 \mu \mathrm{L}$ & $1 \mathrm{U}$ & $0.7 \mu \mathrm{L}$ \\
Calf heart & $9 \mathrm{U}$ & $10 \mu \mathrm{L}$ & $1 \mathrm{U}$ & $0.7 \mu \mathrm{L}$ \\
liver & $8 \mathrm{U}$ & $10 \mu \mathrm{L}$ & $2 \mathrm{U}$ & $1.5 \mu \mathrm{L}$ \\
kidney & $9 \mathrm{U}$ & $10 \mu \mathrm{L}$ & $1 \mathrm{U}$ & $0.7 \mu \mathrm{L}$ \\
Beef heart & $8 \mathrm{U}$ & $10 \mu \mathrm{L}$ & $2 \mathrm{U}$ & $1.5 \mu \mathrm{L}$ \\
kidney & $36 \mathrm{U}$ & $10 \mu \mathrm{L}$ & $4 \mathrm{U}$ & $2.9 \mu \mathrm{L}$ \\
Chicken heart & $8 \mathrm{U}$ & $10 \mu \mathrm{L}$ & $2 \mathrm{U}$ & $1.5 \mu \mathrm{L}$ \\
liver & $8 \mathrm{U}$ & $10 \mu \mathrm{L}$ & $2 \mathrm{U}$ & $1.5 \mu \mathrm{L}$ \\
kidney & $4 \mathrm{U}$ & $10 \mu \mathrm{L}$ & $6 \mathrm{U}$ & $4.4 \mu \mathrm{L}$ \\
stomach & $10 \mathrm{U}$ & $10 \mu \mathrm{L}$ & $10 \mathrm{U}$ & $7.3 \mu \mathrm{L}$ \\
Pig heart & $8 \mathrm{U}$ & $10 \mu \mathrm{L}$ & $2 \mathrm{U}$ & $1.5 \mu \mathrm{L}$ \\
kidney & $3 \mathrm{U}$ & $10 \mu \mathrm{L}$ & $3 \mathrm{U}$ & $2.2 \mu \mathrm{L}$ \\
\hline
\end{tabular}

